\title{
Comparison of Two Mosquito Samples in Resistance Monitoring to Permethrin in Malaria Vectors from Mono Department in South-Western Benin, West Africa
}

\author{
Nazaire Aizoun ${ }^{1,2 *}$ and Luc Djogbénou ${ }^{1,3}$
}

${ }^{1}$ Centre de Recherche Entomologique de Cotonou (CREC), 06 BP 2604, Cotonou, Bénin

${ }^{2}$ Département des Sciences et Techniques Agricoles, Ecole Normale Supérieure de

l'Enseignement Technique (ENSET) de Lokossa, Université Nationale des Sciences,

Technologies, Ingénierie et Mathématiques (UNSTIM) d'Abomey, Bénin

${ }^{3}$ Institut Régional de Santé Publique (IRSP) de Ouidah, Université d'Abomey-Calavi

(UAC), Bénin

*Corresponding author

\begin{tabular}{|c|c|}
\hline & A B S T R A C T \\
\hline & $\begin{array}{l}\text { Pyrethroids are the only group of insecticides currently recommended for net treatment. } \\
\text { Resistance monitoring is essential to investigate the susceptibility of wild populations of } \\
\text { An. gambiae s.l. to pyrethroids. We investigated the kind of mosquito sample useful in the } \\
\text { assessment of insecticide susceptibility tests in malaria vectors in the field conditions. Two }\end{array}$ \\
\hline Keywords & $\begin{array}{l}\text { mosquito samples were used. The first sample concerned larvae and pupae collected using } \\
\text { the dipping method on several breeding sites and the second sample concerned female } A n \text {. }\end{array}$ \\
\hline $\begin{array}{l}\text { Mosquito samples, } \\
\text { Window traps, } \\
\text { Permethrin, WHO } \\
\text { bioassay, CDC bioassay, } \\
\text { Benin }\end{array}$ & $\begin{array}{l}\text { gambiae s.l. mosquitoes collected from window traps put on windows of rooms in districts } \\
\text { surveyed. female An. gambiae s.l. mosquitoes were collected from March-July and } \\
\text { August-November } 2014 \text { during the rainy season in Grand Popo, Comè, Lokossa districts } \\
\text { selected in south-western Benin. WHO bioassays were performed with impregnated papers }\end{array}$ \\
\hline Article Info & of permethrin $0.75 \%$ with the first sample whereas CDC bioassays were performed with \\
\hline $\begin{array}{l}\text { Accepted: } \\
\text { 24 September } 2018 \\
\text { Available Online: } \\
10 \text { October } 2018\end{array}$ & $\begin{array}{l}\text { showed that although two different mosquito samples were used with two different } \\
\text { protocols for the determination of insecticide susceptibility in malaria vectors, the } \\
\text { susceptibility status to permethrin recorded with WHO and CDC methods were the same. } \\
\text { Otherwise, female An. gambiae s.l. populations from Grand Popo, Comè, Lokossa were }\end{array}$ \\
\hline & $\begin{array}{l}\text { resistant to permetrhin. However, the use of female An. gambiae s.l. mosquitoes collected } \\
\text { using the dipping method on several breeding sites may be better than using samples from } \\
\text { window traps in the assessment of insecticide susceptibility tests in malaria vectors in field } \\
\text { conditions. }\end{array}$ \\
\hline
\end{tabular}

\section{Introduction}

Malaria is a major public health problem and Anopheles gambiae is one of the major vectors of this disease in sub-Saharan Africa (Gillies and Coetzee, 1987). Anopheles gambiae Giles (Diptera: Culicidae) is so the major malaria vector in West Africa. Resistance monitoring 
is essential to investigate the susceptibility of wild populations of An. gambiae s.l. to pyrethroids.

Pyrethroids are the only group of insecticides currently recommended for net treatment. The determination of insecticide susceptibility status of the target vectors will help monitor the insecticidal efficacy and possible development of resistance at early stages. So, the early detection of insecticide resistance development is the most important aspect that guides vector control programmes. More recently, the emergence of resistance in populations of An. gambiae to common classes of insecticides used in public health has been reported in Benin (Djogbénou et al., 2008; 2009; Djègbé et al., 2011; Aizoun et al., 2013a; 2013b; 2013c; 2014a; 2014b; 2014c; 2014d; 2014e, 2014f; 2014g; 2014h; 2014i; 2014j).

The ongoing spread of insecticide-resistant genes, such as the well-characterized $k d r$ mutations (Martinez-Torres et al., 1998; Ranson et al., 2000) in populations of the major African malaria vectors, An. gambiae, can seriously jeopardize the efficacy of vector control programmes (Aizoun et al., 2014a). Metabolic resistance or biochemical mechanisms that involve the detoxifying enzymes was also involved in resistance of An. gambiae s.l. populations from Benin (Aizoun et al., 2013a; 2013b; 2014b; 2014c; 2014d).

OlysetNet distribution was made free in July, 2011 throughout the entire country including Mono department by Beninese National Malaria Control Programme to increase coverage of long-lasting insecticidal nets (LLINs). So, people living in areas targeted for the distribution of these long-lasting insecticidal nets, OlysetNet had received them. The insecticide susceptibility status is major factor determining what insecticides should be used in the control campaigns and for that, its determination needs mosquito sample.

The aim of this study is to investigate the kind of mosquito sample useful in the assessment of insecticide susceptibility tests in malaria vectors in the field conditions.

\section{Materials and Methods}

\section{Study area}

The study area is located in Republic of Benin (West Africa) and includes the department of Mono. Mono department is located in the south-western Benin and the study was carried out more precisely in Grand Popo, Comè, Lokossa districts. The choice of the study site took into account the economic activities of populations, their usual protection practices against mosquito bites, and peasant practices to control farming pests. These factors have an impact on resistance development in the local vector mosquitoes. We took them into account to compare both mosquito samples with regard to the resistance level. Mono has a climate with four seasons, two rainy seasons (MarchJuly and August-November) and two dry seasons (November-March and July-August). The temperature ranges from 25 to $30^{\circ} \mathrm{C}$ with the annual mean rainfall between 900 and $1100 \mathrm{~mm}$.

\section{Mosquito sampling}

The first sample concerned An. gambiae s.l. mosquitoes collected from March-July and August-November 2014 during the rainy season in Grand Popo, Comè, Lokossa districts selected in south-western Benin. Larvae and pupae were collected in these districts within both padding and town using the dipping method on several breeding sites (brick pits, pools, marshes, streams, ditches, pits dug for plastering traditional huts, puddles 
of water, water pockets caused by the gutters). Once, larvae and pupae collected, they were then kept in labeled bottles related to the districts surveyed. Otherwise, larvae collected from multiple breeding sites were pooled together then re-distributed evenly in development trays containing tap water. Larvae were provided access to powdered TetraFin ${ }^{\circledR}$ fish food, and were reared to adults under insectary conditions of $25+/-2^{\circ} \mathrm{C}$ and 70 to $80 \%$ relative humidity at Centre de Recherche Entomologique de Cotonou (CREC) located in Akpakpa, in Cotonou district. These samples were reared up to adult emergence at the CREC insectary. $A n$. gambiae Kisumu, a reference susceptible strain was used as a control for the bioassay tests. We used Kisumu more precisely to confirm the quality of treated or impregnated papers. Susceptibility tests were done following WHO protocol on unfed females mosquitoes aged 2-5 days old reared from larval and pupal collections. All WHO susceptibility tests were conducted in the CREC laboratory at $25+/-2^{\circ} \mathrm{C}$ and 70 to $80 \%$ relative humidity.

The second sample concerned An. gambiae s.l. mosquitoes collected in window traps put on windows of four rooms in each district surveyed. Between 6.00 a.m. to 7.00 a.m., aspirators were used to collecting mosquitoes from these window traps. They were then put in some plastic cups covered with small cutting untreated net on which was put cotton wool moistened with a $10 \%$ honey solution. Female An. gambiae species were morphologically identified using morphological keys (Gillies and De Meillon, 1968) and then transferred into mosquito cages. An. gambiae Kisumu, a reference susceptible strain was carried out from Centre de Recherche Entomologique de Cotonou (CREC) insectary to mono department and used as a control for the bioassay tests. We used Kisumu more precisely to confirm the quality of treated Wheaton bottles. All Susceptibility tests were done following CDC protocol on unfed female An. gambiae s.l. mosquitoes.

\section{Testing insecticide susceptibility}

\section{WHO Protocol}

Females An. gambiae s.l. aged 2 to 5 days old were exposed to $\mathrm{WHO}$ diagnostic dosage of permethrin $0.75 \%$ according to the WHO protocol (WHO, 2013). Thus, an aspirator was used to introduce 20 to 25 unfed female mosquitoes into five WHO holding tubes (four tests and one control) that contained untreated papers. They were then gently blown into the exposure tubes containing the insecticide impregnated papers. After one-hour exposure, mosquitoes were transferred back into holding tubes and provided with cotton wool moistened with a $10 \%$ honey solution. The number of mosquitoes "knocked down" at 10 , 20, 30, 40, 50, 60 minutes and mortalities at 24 hours were recorded following the WHO protocol (WHO, 2013). The choice of permethrin was justified by OlysetNet distribution made free in July, 2011 throughout the entire country by Beninese National Malaria Control Programme to increase coverage of long-lasting insecticidal nets (LLINs).

\section{CDC protocol}

The principle of CDC bottle bioassay is to determine the time it takes an insecticide to penetrate an arthropod, traverse its intervening tissues, get to the target site, and act on that site relative to a susceptible control. Anything that prevents or delays the compound from achieving its objective of killing the arthropods contributes to resistance. Diagnostic dose that was applied in the current study was the dose recommended by CDC (Brogdon and Chan, 2010). These doses were 
checked on the An. gambiae Kisumu susceptible reference strain before being applied to field populations. For An. gambiae s.l., the diagnostic dose of $21.5 \mu \mathrm{g}$ per bottle for permethrin was used for a diagnostic exposure time of 30 minutes. The solution was prepared and the bottles coated according to the CDC protocol (Brogdon and Chan, 2010).

Then, these coated Wheaton bottles was carried out from Centre de Recherche Entomologique de Cotonou (CREC) laboratory to mono department. Fifteen to 20 unfed female An. gambiae s.l. mosquitoes were introduced into four $250 \mathrm{ml}$ Wheaton bottles coated with permethrin and one control bottle coated with acetone only.

The number of dead or alive mosquitoes was monitored at different time intervals $(15,30$, $35,40,45,60,75,90,105,120$ minutes). This allowed us to determine the total percent mortality ( $\mathrm{Y}$ axis) against time ( $\mathrm{X}$ axis) for all replicates using a linear scale.

\section{Statistical analysis}

The resistance status of the first mosquito sample was determined according to the latest WHO criteria (WHO, 2013) as follows:

Mortality rates between 98\%-100\% indicate full susceptibility

Mortality rates between $90 \%-97 \%$ require further investigation

Mortality rates < 90\%, the population is considered resistant to the tested insecticides

The resistance status of the second mosquito sample was determined according to the CDC criteria (Brogdon and McAllister, 1998; Brogdon and Chan, 2010). The susceptibility threshold at the diagnostic time of 30 minutes for pyrethroids is:
Mortality rate $=100 \%$ : the population is fully susceptible

Mortality rate < 100\%: the population is considered resistant to the tested insecticides

Abbott's formula was not used in this study for the correction of mortality rates in either the test-tubes or test-bottles because the mortality rates in all controls was always less than 5\% (Abbott, 1987). Analysis using Fisher's exact test and test of proportion was performed on the data sets gathered from the localities surveyed to compare the tested mosquito samples and assess the insecticide resistance status of each tested An. gambiae population using WHO method for the first sample and CDC method for the second sample.

\section{Results and Discussion}

The analysis of table 1 showed that all female mosquitoes of Anopheles gambiae Kisumu which were exposed to WHO papers impregnated with permethrin $0.75 \%$ were knocked-down after 30 minutes whereas a non-neglected proportion of An. gambiae s.l. Grand Popo, Comè and Lokossa populations; $62.9 \%, 54.5 \%$ and $85.7 \%$ respectively continue again to fly in the WHO cylinder plastic tubes which contained these impregnated papers. The analysis of table 2 showed that after 24 hours mortality recording Kisumu strain (control) confirmed its susceptibility status as a reference strain whereas An. gambiae s.l. Grand Popo, Comè and Lokossa populations were resistant to permethrin $0.75 \%$. According to Zaim et al., (2000), pyrethroids have unique modes of action such as fast knockdown and excitorepellent effects.

In the same way, the analysis of table 1 shows that all female mosquitoes of Anopheles gambiae Kisumu which were exposed to CDC 
bottles treated with permethrin $21.5 \mu \mathrm{g} / \mathrm{bottle}$ were died after 30 minutes, which represents susceptibility threshold time or diagnostic time clearly defined by CDC protocol. That showed Kisumu strain (control) confirmed its susceptibility status as a reference strain. A non-neglected proportion of An. gambiae s.l. Grand Popo, Comè and Lokossa populations; $13,3 \%, 15 \%$ and $11,1 \%$ respectively after 30 minutes exposure to CDC bottles treated with permethrin, continue again to fly in these bottles. That showed these populations were resistant to this product.

Although two different mosquito samples were used with two different protocols for the determination of insecticide susceptibility in malaria vectors, the susceptibility status to permethrin recorded with WHO and CDC methods were the same (Table 2). The mortality rates recorded with CDC method were slightly higher than those obtained with WHO method (Table 2). This slight increase should be explained by the age of the second sample used. Otherwise, the age of mosquitoes which entered the rooms by the doors or others windows where traps were not put, was not known. Then, these mosquitoes entered the window traps by going out through windows early in the morning.

They were likely old mosquitoes. In fact, a previous study by Aizoun et al., (2014k) showed that the mortality rate obtained when female An. gambiae s.l. Sekandji populations were unfed and aged 20 days old was higher than the one obtained when these populations were unfed and aged 2-5 days old.

Table.1 Knock-down at 30 minutes with WHO method and mortality at the susceptibility threshold with CDC method

\begin{tabular}{|l|c|c|c|c|c||}
\hline & \multicolumn{4}{|c|}{ Permethrin } & \\
\hline & $\begin{array}{c}\text { Number } \\
\text { tested }\end{array}$ & $\begin{array}{l}\text { \%d at } \\
30 \mathrm{~min}\end{array}$ & $\begin{array}{c}\text { Number } \\
\text { tested }\end{array}$ & $\begin{array}{l}\text { \% Mortality at } \\
30 \mathrm{~min}\end{array}$ \\
\hline $\begin{array}{l}\text { Kisumu } \\
\text { (Control) }\end{array}$ & 100 & 100 & 100 & 100 \\
\hline Grand Popo & 100 & 37.1 & 19 & 86.7 \\
\hline Comè & 100 & 45.5 & 22 & 85 \\
\hline Lokossa & 100 & 14.3 & 14 & 88.9 \\
\hline
\end{tabular}

Table.2 Susceptibility status to permethrin in Anopheles gambiae s.l. populations

\begin{tabular}{|l|c|c|c|c|c|c|c|}
\hline & \multicolumn{9}{|c|}{ WHO } & \multicolumn{2}{c|}{ CDC } & WHO & CDC \\
\hline Populations & $\begin{array}{c}\text { Number } \\
\text { tested }\end{array}$ & $\begin{array}{c}\text { Mortality } \\
\text { Mumber }\end{array}$ & $\begin{array}{c}\text { \% } \\
\text { tested } \\
\text { Mortality }\end{array}$ & $\begin{array}{l}\text { Resistance } \\
\text { status }\end{array}$ & $\begin{array}{l}\text { Resistance } \\
\text { status }\end{array}$ \\
\hline $\begin{array}{l}\text { Kisumu } \\
\text { (Control) }\end{array}$ & 100 & 100 & 100 & 100 & S & S \\
\hline Grand Popo & 100 & 73.7 & 19 & 86.7 & R & R \\
\hline Comè & 100 & 72.7 & 22 & 85 & R & R \\
\hline Lokossa & 100 & 78.6 & 14 & 88.9 & R & R \\
\hline
\end{tabular}


A previous study which used the same mosquito sample or female An. gambiae s.l. mosquitoes collected by using the dipping method on several breeding sites, with both WHO and CDC methods also showed the same susceptibility status to insecticides (Aizoun et al., 2013a). The age of tested female An. gambiae s.l. mosquitoes collected in the window traps put on windows of four rooms in each district surveyed was not known whereas the age of tested female $A n$. gambiae s.l. mosquitoes collected by using the dipping method on several breeding sites (brick pits, pools, marshes, streams, ditches, pits dug for plastering traditional huts, puddles of water, water pockets caused by the gutters) was known. But, that had no impact on susceptibility results recorded with both methods in the current study. However, Aizoun et al., (2014k), showed that many factors influence vector susceptibility to insecticide. Among these factors, there are mosquito sex, physiological status and mosquito age. That is for this reason, it is useful to respect the WHO criteria in the assessment of insecticide susceptibility tests in malaria vectors. Otherwise, susceptibility testing is conducted using unfed female mosquitoes aged 3-5 days old. Tests should also be carried out at $(25+/-2){ }^{\circ} \mathrm{C}$ and $(80+/-$ $10) \%$ relative humidity $((\mathrm{WHO}, 2013)$. In the current study, the temperature recorded during the assessment of insecticide susceptibility tests on the field or in Mono department ranged from 25 to $30^{\circ} \mathrm{C}$ whereas the relative humidity ranged from 70 to $80 \%$. Sometimes, when the insectary is very far from the field or localities surveyed, it is useful that the collected larvae and pupae are reared up to adult emergence on the field but that is not easy because of field conditions. This way to process may be better than using samples from window traps. That also depend on the number of consecutive days the surveys take on the field. For that, Aizoun et al., (20141), had studied the efficacy of a
WHO impregnated paper with bendiocarb in field conditions.

The current study shows that mosquito samples from dipping method on several breeding sites or larvae and pupae collections and those from the window traps put on windows of rooms in districts surveyed gave similar results with regard to susceptibility status to permethrin recorded with both WHO and CDC methods. However, the use of the mosquito samples from larvae and pupae collections may be better than using samples from window traps in the assessment of insecticide susceptibility tests in malaria vectors in field conditions.

\section{Acknowledgements}

We are grateful to the Ministère de l'Enseignement Supérieur et de la Recherche Scientifique (MESRS) of Benin which financially supported this study and the doctoral training of Nazaire. We would like to thank Dr William G. BROGDON from CDC Atlanta, USA who supplied us the reagents used for CDC bioassays. The authors would also like to thank Frederic OKE-AGBO for statistical analysis. Tel: (229) 95317939.

\section{References}

Abbott, W.S. 1987. A method of computing the effectiveness of an insecticide. J. Am. Mosq. Control. Assoc., 3(2):302-303.

Aïzoun, N., Aïkpon, R., Akogbéto, M. 2014a. Evidence of increasing L1014F $k d r$ mutation frequency in Anopheles gambiae s.l. pyrethroid resistance following a nationwide distribution of LLINs by the Beninese National Malaria Control Programme. Asian. Pac. J. Trop. Biomed., 4 (3):239-243.

Aïzoun, N., Aïkon, R., Azondekon, R., Asidi, A., Akogbéto, M. 2014k. Comparative susceptibility to permethrin of two Anopheles gambiae s.l. populations from 
southern Benin, regarding mosquito sex, physiological status, and mosquito age. Asian. Pac. J. Trop. Biomed., 4(4):312317.

Aïzoun, N., Aïkpon, R., Gnanguenon, V., Azondekon, R., Oké-Agbo, F., Padonou, G.G., Akogbéto, M. 2014b. Dynamics of insecticide resistance and effect of synergists piperonyl butoxide (PBO), S.S.S-tributylphosphorotrithioate (DEF) and ethacrynic acid (ETAA or EA) on permethrin, deltamethrin and dichlorodiphenyltrichloroethane (DDT) resistance in two Anopheles gambiae s.l. populations from southern Benin, West Africa. J. Parasitol. Vector. Biol., 6(1):110.

Aïzoun, N., Aïkpon, R., Gnanguenon, V., Oussou, O., Agossa, F., Padonou, G.G., Akogbéto, M. 2013c. Status of organophosphate and carbamate resistance in Anopheles gambiae sensu lato from the south and north Benin, West Africa. Parasit Vectors, 6:274.

Aïzoun, N., Aïkpon, R., Padonou, G.G., Oussou, O., Oké-Agbo, F., Gnanguenon, V., Ossè, R., Akogbéto, M. 2013b. Mixed-function oxidases and esterases associated with permethrin, deltamethrin and bendiocarb resistance in Anopheles gambiae s.l. in the south-north transect Benin, West Africa. Parasit Vectors, 6:223.

Aïzoun, N., Azondekon, R. and Akogbéto, M. 2014d. Exploring Glutathione $S$ transferases involved in dichlorodiphenyltrichloroethane (DDT) and permethrin cross-resistance in Anopheles gambiae s.l. populations in the south-north transect Benin, West Africa. Int. J. Curr. Microbiol. App. Sci., 3(9): 392-403.

Aïzoun, N., Azondekon, R. and Akogbéto, M. 2014e. The L1014F $k d r$ mutation in Anopheles gambiae s.l. lambdacyhalothrin resistant populations from Kandi district in northen Benin, West Africa. Int. J. Curr. Res. Biosci. Plant. Biol., 1(4): 9-14.
Aïzoun, N., Azondekon, R. and Akogbéto, M. $2014 \mathrm{j}$. Susceptibility status to malathion in Anopheles gambiae s.l. populations from Toffo district in southern Benin, West Africa. Int. J. Curr. Microbiol. App. Sci., 3(10): 893-898.

Aïzoun, N., Azondekon, R., Aïkpon, R., Anagonou, R., Gnanguenon, V., and Akogbéto, M. 2014c. Dynamics of insecticide resistance and exploring biochemical mechanisms involved in pyrethroids and dichlorodiphenyltrichloroethane (DDT) cross-resistance in Anopheles gambiae s.l. populations from Benin, West Africa. $J$. Cell. Anim. Biol., 8(3): 41-50.

Aïzoun, N., Azondekon, R., Aïkpon, R., Gnanguenon, V., Ossè, R., Asidi, A., Akogbéto, M. 2014l. Study of the efficacy of a Wheaton coated bottle with permethrin and deltamethrin in laboratory conditions and a WHO impregnated paper with bendiocarb in field conditions. Asian. Pac. J. Trop. Biomed., 4(6):492497.

Aïzoun, N., Azondekon, R., and Akogbéto, M. 2014g. Status of deltamethrin resistance in three Anopheles gambiae sensu lato populations from main ecological settings in Benin, West Africa. Int. J. Curr. Microbiol. App. Sci., 3(9): 58-64.

Aïzoun, N., Azondekon, R., and Akogbéto, M. 2014h. Propoxur resistance in Anopheles gambiae s.l. populations from N'dali district in northern Benin, West Africa. Int. J. Curr. Microbiol. App. Sci., 3(9): 224-229.

Aïzoun, N., Azondekon, R., and Akogbéto, M. 2014i. Cyfluthrin resistance in Anopheles gambiae s.l. populations from the cotton growing area of Hlassoé in Glazoué district in the central part of Benin, West Africa. Int. J. Curr. Microbiol. App. Sci., 3(10): 899-904.

Aïzoun, N., Gnanguenon, V., Azondekon, R., Anagonou, R., Aïkpon, R., Akogbéto, M. 2014f Status of organophosphate and carbamate resistance in Anopheles gambiae sensu lato from the Sudano 
Guinean area in the central part of Benin, West Africa. J. Cell. Anim. Biol., 8(4):6168.

Aïzoun, N., Ossè, R., Azondekon, R., Alia, R., Oussou, O., Gnanguenon, V., Aikpon, R., Padonou, G.G., Akogbéto, M. 2013a. Comparison of the standard WHO susceptibility tests and the CDC bottle bioassay for the determination of insecticide susceptibility in malaria vectors and their correlation with biochemical and molecular biology assays in Benin, West Africa. Parasit Vectors, 6: 147.

Brogdon, W., Chan, A. 2010. Guidelines for Evaluating Insecticide Resistance in Vectors using the CDC Bottle Bioassay/ Methods in anopheles research. Second edition. CDC Atlanta USA: CDC technical report; 2010:343.

Brogdon, W.G., McAllister, J.C.1998. Simplification of adult mosquito bioassays through use of time-mortality determinations in glass bottles. J. Am. Mosq. Control. Assoc., 14(2):159-164.

Djègbé, I., Boussari, O., Sidick, A., Martin, T., Ranson, H., Candre, F., Akogbéto, M., Corbel, V. 2011. Dynamics of insecticide resistance in malaria vectors in Benin: first evidence of the presence of L1014S $k d r$ mutation in Anopheles gambiae from West Africa. Malar. J., 10:261.

Djogbenou, L., Dabire, R., Diabate, A., Kengne, P., Akogbeto, M., Hougard, J.M., Chandre, F. 2008. Identification and geographic distribution of the ACE-1R mutation in the malaria vector Anopheles gambiae in south-western Burkina Faso,
West Africa. Am. J. Trop. Med. Hyg., 78:298-302.

Djogbenou, L., Pasteur, N., Akogbeto, M., Weill, M., Chandre, F. 2009. Insecticide resistance in the Anopheles gambiae complex in Benin: a nationwide survey. Med. Vet. Entomology., 69: 160-164.

Gillies, M.T., Coetzee, M. 1987. A supplement to the Anophelinae Africa South of the Sahara (Afrotropical region), Johannesburg, South Africa. S. Afr. Inst. Med. Res., 55: 1-143.

Gillies, M.T., De Meillon, B. 1968. The Anophelinae of Africa south of the Sahara Publication of the South African Institute for Medical Research, Johannesburg; 1968:54.

Martinez-Torres, D., Chandre, F., Williamson, M.S., Darriet, F., Berge, J.B., Devonshire, A.L., Guillet, P., Pasteur, N., Pauron, D.1998. Molecular characterization of pyrethroid knockdown resistance $(k d r)$ in major malaria vector An. gambiae s.s. Insect. Mol. Bio., 7, 179-184.

Ranson, H., Jensen, B., Vulule, J.M., Wang, X., Hemingway, J., Collins, F.H. 2000. Identification of a point mutation in the voltage-gated sodium channel gene of Kenyan Anopheles gambiae associated with resistance to DDT and pyrethroids. Insect. Mol. Biol., 9(5), 491-497.

WHO. 2013. Test procedures for insecticide resistance monitoring in malaria vector mosquitoes. Geneva, World Health Organization.

Zaim, M., Aitio, A., Nakashima, N. 2000. Safety of pyrethroid-treated mosquito nets. Med. Vet. Entomol., 14:1-5.

\section{How to cite this article:}

Nazaire Aizoun and Luc Djogbénou. 2018. Comparison of Two Mosquito Samples in Resistance Monitoring to Permethrin in Malaria Vectors from Mono Department in South-Western Benin, West Africa. Int.J.Curr.Microbiol.App.Sci. 7(10): 3270-3277. doi: https://doi.org/10.20546/ijcmas.2018.710.378 